\title{
AN ORIENTED MODEL FOR KHOVANOV HOMOLOGY
}

\author{
CHRISTIAN BLANCHET
}

\begin{abstract}
We give an alternative presentation of Khovanov homology of links. The original construction rests on the Kauffman bracket model for the Jones polynomial, and the generators for the complex are enhanced Kauffman states. Here we use an oriented $s l(2)$ state model allowing a natural definition of the boundary operator as twisted action of morphisms belonging to a TQFT for trivalent graphs and surfaces. Functoriality in original Khovanov homology holds up to sign. Variants of Khovanov homology fixing functoriality were obtained by Clark-Morrison-Walker [CMW] and also by Carmen Caprau [CC]. Our construction is similar to those variants. Here we work over integers, while the previous constructions were over gaussian integers, and produce the TQFT by a universal construction. We consider diagrams in the oriented plane. Our functoriality results include that for a fixed link the homology isomorphism associated with a sequence of Reidemeister moves between two diagrams is canonical.
\end{abstract}

\section{Trivalent TQFT}

1.1. Frobenius algebra. TQFTs for oriented surfaces are in one to one correspondence with commutative Frobenius algebras (also called symmetric algebras) $\left[\mathrm{Ko}\right.$. We consider here the Frobenius algebra $\mathbf{A}=\mathbb{Z}[X] / X^{2} \approx H^{*}\left(\mathbb{C} P^{1}\right)$, and we denote by $V_{\mathbf{A}}$ the associated TQFT. The unit element in $\mathbf{A}$ is denoted by $\mathbf{1}$; the coalgebra structure $(\Delta, \epsilon)$ on $\mathbf{A}$ is defined by

$$
\begin{gathered}
\epsilon(X)=1, \epsilon(\mathbf{1})=0 ; \\
\Delta(X)=X \otimes X, \Delta(\mathbf{1})=\mathbf{1} \otimes X+X \otimes \mathbf{1} .
\end{gathered}
$$

The invariant of a closed surface is given below.

$$
V_{\mathbf{A}}\left(S^{1} \times S^{1}\right)=\operatorname{rank}(\mathbf{A})=2,
$$

$V_{\mathbf{A}}\left(\Sigma_{g}\right)=0$ for a closed surface $\Sigma_{g}$ with genus $g \neq 1$.

The TQFT is extended to surfaces with points. The neighbourhood of a point represents the element $X$ in the algebra associated with the oriented circle $V_{\mathbf{A}}\left(S^{1}\right)=\mathbf{A}$. For a genus $g$ closed surface with $k$ points the invariant is zero excepted

for $(g, k)=(1,0)$ where the value is 2 , and

for $(g, k)=(0,1)$ where the value is 1 .

1.2. The universal construction. One can reconstruct the above TQFT from the invariant of closed surfaces with points. The TQFT module of an oriented curve $\gamma$ is generated over $\mathbb{Z}$ by surfaces with points whose boundary is identified with $\gamma$. Relations are given by the kernel of the bilinear form defined by gluing. A key point in proving that the functor $V_{A}$ defined this way is indeed a TQFT is the surgery formula in Figure 1 .

Here cobordisms are depicted from left to right. The graphical identity can be 


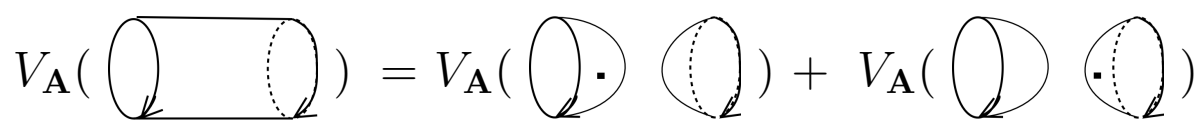

Figure 1. Surgery formula
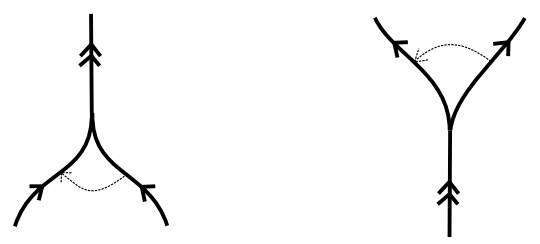

Figure 2. Trivalent vertices

written

$$
\operatorname{Id}_{\mathbf{A}}=\epsilon(X \times \cdot) \mathbf{1}+\epsilon(\cdot) X
$$

1.3. Graded TQFT. We define a grading on $\mathbf{A}=\mathbb{Z}[X] / X^{2}$, by $\operatorname{deg}(\mathbf{1})=1$, $\operatorname{deg}(X)=-1$. The $q$-dimension (or Poincaré polynomial) of the TQFT module associated with a $k$ components curve is $\left(q+q^{-1}\right)^{k}$. The TQFT functor is graded. For a cobordism $\Sigma$ between $\gamma$ and $\gamma^{\prime}$, the linear map

$$
V_{\mathbf{A}}(\Sigma): V_{\mathbf{A}}(\gamma) \rightarrow V_{\mathbf{A}}\left(\gamma^{\prime}\right)
$$

has degree $\chi(\Sigma)-2 \sharp$ pts. Here $\chi(\Sigma)$ is the Euler characteristic, and $\sharp$ pts is the number of points.

1.4. Trivalent category. We will extend the TQFT over the cobordism category whose objects are trivalent graphs and whose morphisms are trivalent surfaces. Here a trivalent graph is an oriented graph with edges labelled with 1 or 2 , and 3 -valent vertices where the flow condition is respected. For each trivalent vertex an order on the 2 (germs of) edges labelled with 1 is fixed. For a planar graph we use plane orientation and fix the order according to the rule depicted in figure 2 the labels of the edges are obviously encoded in the arrows.

A closed trivalent surface is a 2-complex whose regular faces are oriented and labelled with 1 or 2 . The singular locus is a curve called the binding; each component of the binding has a neighborhood which is a trivalent vertex times $S^{1}$, i.e. there are two 1-labelled pages inducing the same orientation and one 2-labelled page inducing the opposite orientation. For each component of the binding, an order on the two 1-labelled pages is fixed. A 1-labelled face may have points on it.

Cobordisms are obtained by cutting in a generic way. They are considered up to the usual equivalence of oriented homeomorphism rel. boundary.

1.5. TQFT on the trivalent category. The following general procedure constructs a functor (hopefully a TQFT functor) on the trivalent cobordism category. We first define an invariant of closed trivalent surfaces, and extend it into a functor on the trivalent category via the universal construction introduced in [BHMV] and sketched above 1.2 for surfaces.

Suppose that we are given Frobenius algebras $A, B$ and $C$ over a ring $\mathbf{k}$, with corresponding TQFT functors denoted by $V_{A}, V_{B}$ and $V_{C}$. Let $\Sigma$ be a closed 
trivalent surface, and $\dot{\Sigma}=\Sigma_{1} \amalg \Sigma_{2}$ be the surface cut along the binding, decomposed according to the label of the faces. Let $m$ be the number of components of the binding of $\Sigma$. The boundary of $\Sigma_{1}$ has $2 m$ oriented components $C_{i}^{-}$and $C_{i}^{+}$, $1 \leq i \leq m$, and the boundary of $\Sigma_{2}$ has $m$ components $C_{i}^{2}$. Here the $\mp$ is fixed with respect to the ordering of the 1-labelled pages. The TQFT functors $V_{A}$ and $V_{B}$ associate to $\Sigma_{1}$ and $\Sigma_{2}$ vectors

$$
\begin{gathered}
V_{A}\left(\Sigma_{1}\right) \in \bigotimes_{i=1}^{m}\left(V_{A}\left(C_{i}^{-}\right) \otimes V_{A}\left(C_{i}^{+}\right)\right) \cong(A \otimes A)^{\otimes m}, \\
V_{B}\left(\Sigma_{2}\right) \in \bigotimes_{i=1}^{m} V_{B}\left(C_{i}^{2}\right) \cong B^{\otimes m} .
\end{gathered}
$$

Now suppose that we are given maps $f=A \otimes A \rightarrow C, g: B \rightarrow C$, then we define the invariant $\mathbf{V}(\Sigma)$ by the formula

$$
\mathbf{V}(\Sigma)=\left(\epsilon_{C}\right)^{\otimes m}\left(f^{\otimes m}\left(V_{A}\left(\Sigma_{1}\right)\right) \times g^{\otimes m}\left(V_{B}\left(\Sigma_{2}\right)\right) \in \mathbf{k}^{\otimes m}=\mathbf{k} .\right.
$$

Here $\epsilon_{C}: C \rightarrow \mathbf{k}$ is the trace on the Frobenius algebra $C$; the product is computed in $C^{\otimes m}$.

From now on, we use the Frobenius algebras over $\mathbb{Z}: \mathbf{A}=\mathbb{Z}[X] / X^{2} \approx H^{*}\left(\mathbb{C} P^{1}\right)$, $C=\mathbf{A}$, and $B=\mathbb{Z}$ with non standard trace $\epsilon_{C}(n)=-n$. The structural map $f$ is defined by $f(x \otimes y)=x \bar{y}$, where $\overline{a+b X}=a-b X(a, b \in \mathbb{Z})$, and $g: C=\mathbb{Z} \rightarrow \mathbf{A}=B$ is the unit map.

Example 1.1. Let us consider the trivalent surface which is a sphere together with a 2-labelled meridional disk, and whose 1-labelled half-spheres are ordered northsouth. The associated value

is 0 if there is no point,

is 1 if there is one point which is on north half-sphere,

is -1 if there is one point which is on south half-sphere,

is 0 if there is more than one point.

The universal construction extends the invariant $\mathbf{V}$ to a functor on the trivalent cobordism category. The following proposition shows that the functor $\mathbf{V}$ is an extension of the TQFT functor $V_{A}$.

Proposition 1.2. a) We have a natural transformation from $V_{A}$ to $\mathbf{V}$, i.e. for an oriented curve $\gamma$, we have a TQFT module $V_{\mathbf{A}}(\gamma)$, a module $\mathbf{V}(\gamma)$, and a natural map

$$
i_{\gamma}: V_{\mathbf{A}}(\gamma) \rightarrow \mathbf{V}(\gamma)
$$

Here we label all the components of $\gamma$ with 1 , and consider $\gamma$ as an object in the trivalent category.

b) For any curve $\gamma$, the natural map $i_{\gamma}$ is an isomorphism.

Proof. The surgery formula in Figure 1 holds for surgery on a 1-labelled face of a trivalent surface. Using this formula along each component of the curve $\gamma$, we see that any trivalent surface with boundary $\gamma$ representing a generator of $\mathbf{V}(\gamma)$ can be written as a linear combination of disks (may be with points), and that any linear combination representing a relation in $V_{A}(\gamma)$ also represents a relation in $\mathbf{V}(\gamma)$. This proves existence and surjectivity of $i_{\gamma}$. Injectivity of $i_{\gamma}$ and naturality follow from the definitions in the universal construction. 


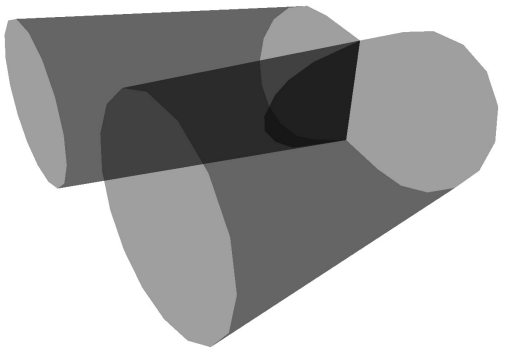

FiguRE 3. Trivalent surface with grading -1

The extended functor $\mathbf{V}$ is still graded. The formula for a cobordism $\Sigma$ is

$$
\operatorname{deg}(\Sigma)=\chi_{1}(\Sigma)-2 \sharp \text { pts . }
$$

Here $\chi_{1}(\Sigma)$ is the Euler characteristic of the 1-labelled subsurface, e.g. the saddle with 2-labelled membrane in figure 3 has grading -1 . Here 1-labelled faces are depicted in light grey and the 2-labelled half-disc is black.

The lemma below gives some examples of computation with the extended functor V. These results will be useful in the subsequent categorification procedure. In the pictures, the order on the germs of 1-labelled edges is fixed by the following plane convention (Figure 2): the first 1-labelled edge is on the right of the oriented 2labelled adjacent edge. Proofs are left as exercise.

Lemma 1.3. a) If $\Sigma^{\prime}$ is obtained from $\Sigma$ by moving a point across a component of the binding then $\mathbf{V}\left(\Sigma^{\prime}\right)=-\mathbf{V}(\Sigma)$.

b) The bubble relations in Figure 4 hold.

c) The band moves relations in Figure 5 hold.

d) The tube relations in Figure 6 hold (the sign depends on the order of the 1-labelled pages at each binding).

In the pictures the 2-labelled faces are depicted in black, the 1-labelled faces are depicted in grey. The small arc indicates the order around the binding.

\section{CAtegorification of the sl(2) invariant of Planar GRAPhs}

We consider here trivalent planar graphs whose edges are smooth; each edge has a label equal to 1 or 2 . In each trivalent vertex, the flow is conserved, and the tangent vectors are coherent. Loops with label 1 are accepted. The admissible vertices are depicted in Figure 2, In the representation theoretic setting, 1-labelled edges correspond to the standard representation of $s l(2)$, and 2-labelled edges correspond to its determinant (isomorphic to the trivial representation).

An enhancement $\epsilon$ for such a graph is a map from the set of 1-labelled edges to $\{-1,1\}$ required to have distinct values for the two edges adjacent to a trivalent vertex. To each trivalent vertex $v$ we associate a weight $\mathcal{W}(v)=q^{ \pm \frac{1}{2}}$. Here $q$ is an indeterminate, and the sign is given by the state of the right handed edge. The $s l(2)$ invariant of such a graph $G$ is given by

$$
\langle G\rangle=\prod_{\text {vertices } v} \mathcal{W}(v) q^{\sum_{a} \epsilon(a) \operatorname{rot}(a)} .
$$



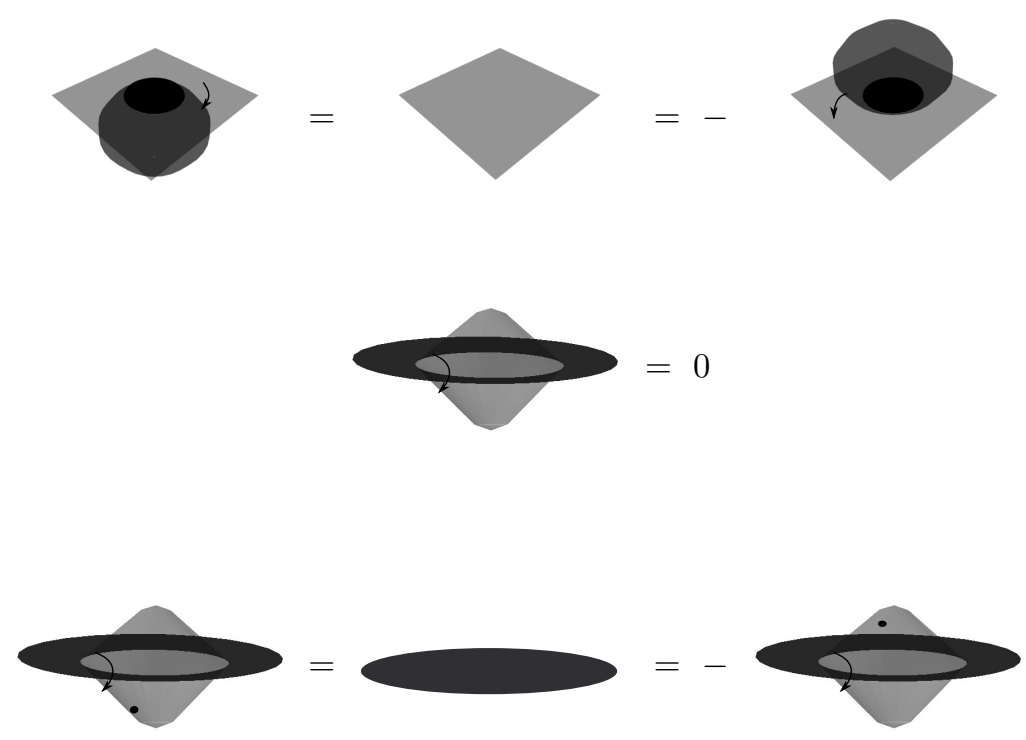

Figure 4. Bubble relations
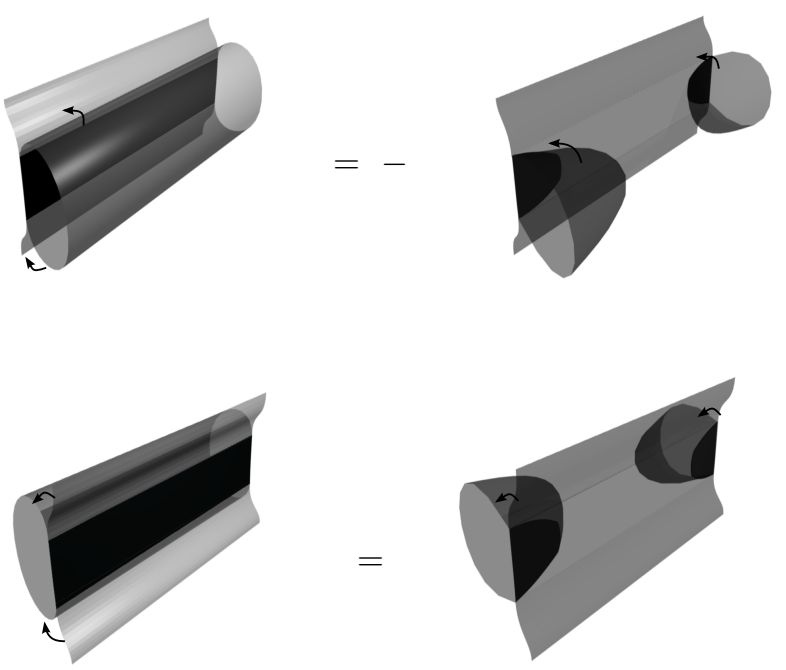

FiguRE 5. Band relations 


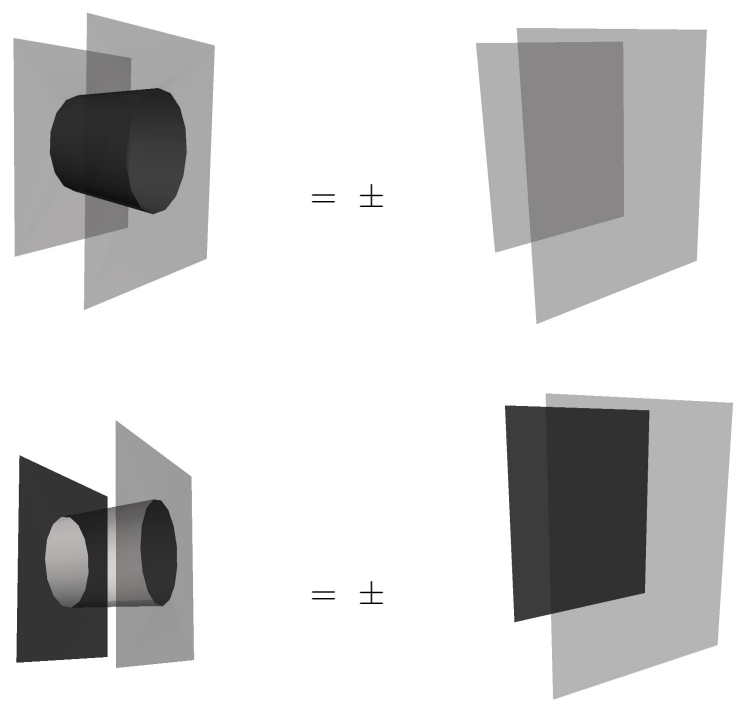

Figure 6. Tube relations

The sum is over all 1-labelled edges $a$, and $\operatorname{rot}(a)$ is the variation of the tangent vector along the edge, normalized so that it gives the Whitney degree (signed number of rotation) for a closed curve.

The invariant $\langle G\rangle$ is easily seen to be equal to $\left(q+q^{-1}\right)^{\sharp G_{1}}$ where $\sharp G_{1}$ is the number of components of the curve composed with the 1-labelled edges. Its interest is that it allows to give a state model for the Jones polynomial similar to the Kauffman bracket state model, but taking into account the orientation.

We associate to such a graph the graded module $\mathbf{V}(G)=\oplus_{k} \mathbf{V}_{k}(G)$. For any graph $G$, the module $\mathbf{V}(G)$ admits a finite set of generators which can be obtained by first pairing the trivalent vertices with singular arcs and then gluing discs, may be with points on the 1-labelled ones. The module itself can then be computed using the pairing.

Exercise 2.1. Let $G_{1}, G_{2}, G 3, G_{4}$ be the graphs depicted in Figure 7. Show that

$$
\mathbf{V}\left(G_{1}\right) \approx \mathbf{A}, \mathbf{V}\left(G_{2}\right) \approx \mathbf{A}^{\otimes 2}, \mathbf{V}\left(G_{3}\right) \approx \mathbf{A}^{\otimes 2}, \mathbf{V}\left(G_{4}\right) \approx \mathbf{A}
$$

Proposition 2.2. $\mathbf{V}(G)$ is a free abelian group whose q-dimension is equal to the invariant $\langle G\rangle$ :

$$
\langle G\rangle=\sum_{k} q^{k} \operatorname{rank}\left(\mathbf{V}_{k}(G)\right) .
$$

Remark 2.3. We understand this theorem as a categorification of the invariant $\langle G\rangle$. Indeed, the functor $\mathbf{V}$ associates to a graph $G$ a graded abelian group which can be interpreted as (co)homology concentrated in (co)homological degree zero. The purpose of the next section is to extend this categorification to link diagrams.

Proof. It is enough to prove the formula for a connected graph. We proceed by induction on the number of 2-labelled edges. If this number is 0 , we get a loop 

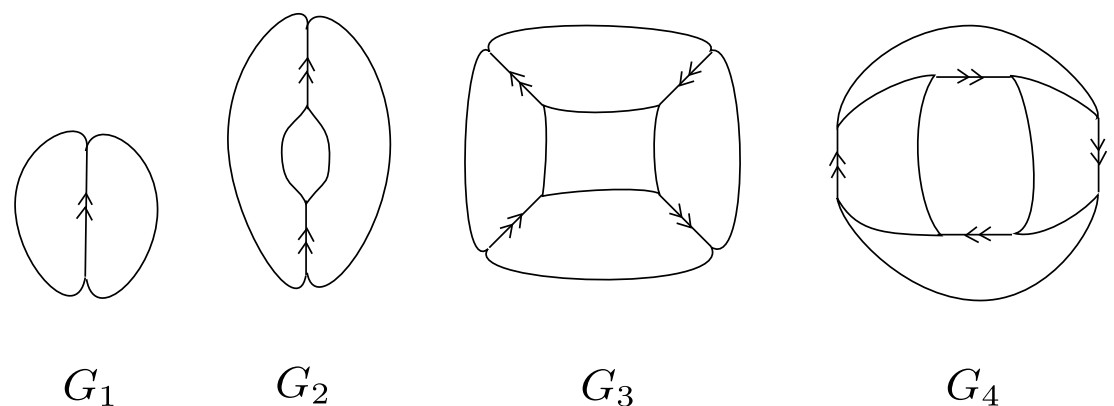

$G_{1}$

$G_{3}$

$G_{4}$

FIGURE 7.
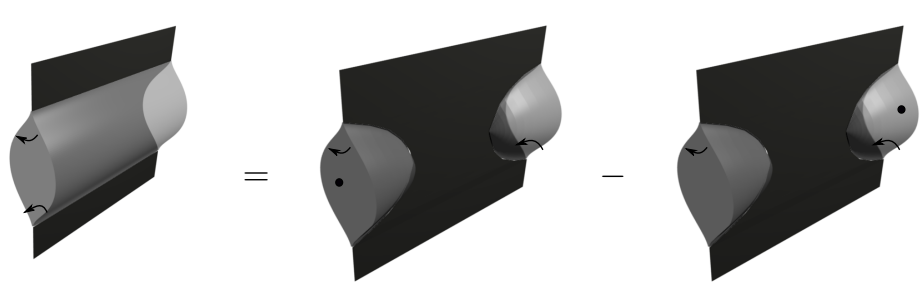

Figure 8. Bigon relation

whose value is $q+q^{-1}$. By an Euler characteristic argument a trivalent graph will have at least one face which is either a bigon or a square. Lemmas 2.4 and 2.6 below shows that the computation reduces to graphs with less 2-labelled edges.

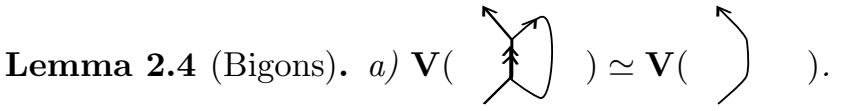

b) $\mathrm{v}(\circlearrowleft) \simeq \mathrm{V}\left(\nearrow^{\prime}\right.$.

c) $\mathbf{V}($

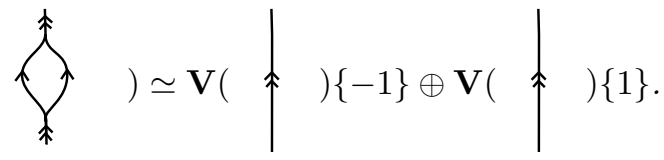

Here the bracket in right hand side of c) indicates a shift in the grading.

Proof. a) and b) are deduced from the band relations in Lemma 1.3 c. Indeed the cobordisms in the right hand side of these relations can be decomposed by cutting in the middle. The induced TQFT maps give the needed isomorphisms.

Lemma 2.5 below, whose proof is left to the reader, decomposes identity of the module on the left hand side of c) into two orthogonal idempotents whence the direct sum decomposition. Note the shift given by the degree of the cobordisms inducing the projection on each summand.

Lemma 2.5. The relations in figure 8 and 9 holds. 


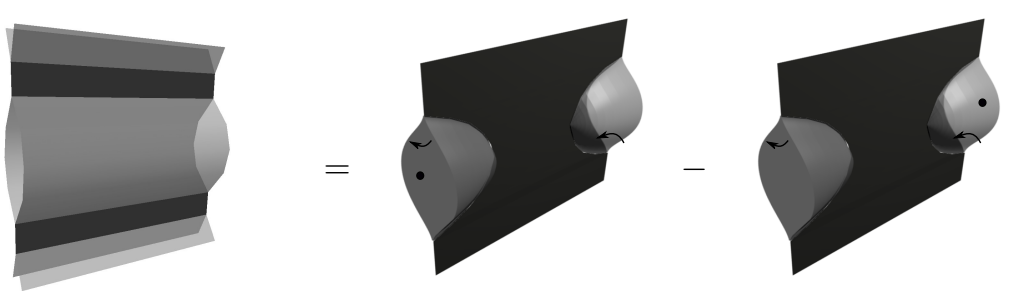

FiguRE 9. 4 terms relation
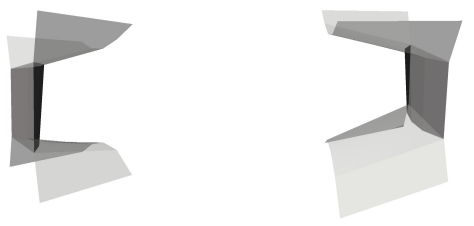

Figure 10. Isomorphisms in Lemma 2.6 a)
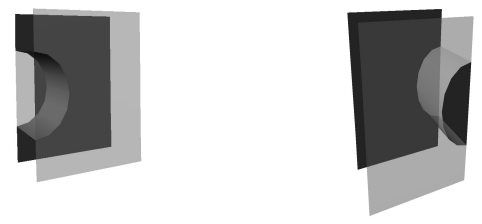

FIgURE 11. Isomorphisms in Lemma 2.6 b)

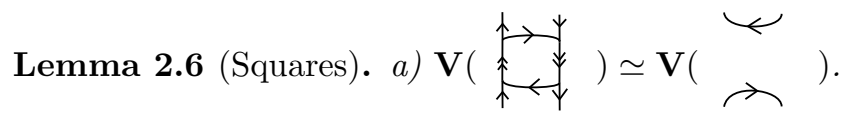
b) $\mathbf{V}(\underset{+}{+}) \simeq \mathrm{V}(\uparrow \uparrow)$.

Proof. Isomorphisms in a) are depicted in figure 10. Both compositions give identity up to sign. One can be seen using relations in Lemma 1.3. Isomorphisms in b) are described in figure 11 .

\section{Khovanov HOMOLOGY}

3.1. Jones polynomial via planar graphs. The formulas below extend the preceeding $s l(2)$ invariant of planar trivalent graphs to an invariant of link diagrams. 


$$
\begin{aligned}
& \left.\langle\Uparrow\rangle=q^{-1}\langle)(\rangle-q^{-2}\right\rangle
\end{aligned}
$$

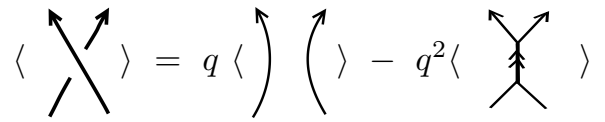

The normalisation for the empty link is 1 , and we have the following skein relation

$$
q^{2}\langle/\rangle-q^{-2}\langle\gg\rangle=\left(q-q^{-1}\right)\langle)(\rangle
$$

Up to normalisation, we recognize the Jones polynomial with the change of variable $q=-t^{-\frac{1}{2}}$. A global state sum formula for a link represented by a diagram $D$ is given below. Note that it is quite easy to show that this formula is invariant under Reidemeister move; this is a slight variant of the Kauffman bracket construction.

We give a global state sum formula for a diagram $D$. A state $s$ of $D$ associates to a positive (resp. negative) crossing either 0 or 1 (resp. -1 or 0 ). For a state $s$, $D_{s}$ is the planar trivalent graph, defined by the rule:

$$
\begin{aligned}
& \text { if } s(c)=0 \text {, then } c \text { is replaced by } \\
& \text { if }|s(c)|=1 \text {, then } c \text { is replaced by }
\end{aligned}
$$

One has

$$
\langle D\rangle=\sum_{s} q^{-(w(D)+s(D))}\left\langle D_{s}\right\rangle
$$

Here $w(D)=\sum_{c} \operatorname{sign}(c)$, and $s(D)=\sum_{c} s(c)$.

3.2. Khovanov complex. We consider a link diagram $D$. For a state $s$, we define the trivalent graph $D_{s}$ according to the local rules described just above. We use the notation $d_{s}$ for $\sum|s(c)|$, and $\Delta_{s}$ for the free abelian group generated by crossings $c$ with $|s(c)|=1$. The Khovanov complex is a bigraded abelian group $K(D)$ defined below, together with a convenient boundary operator.

$$
K(D)=\bigoplus_{s} V\left(D_{s}\right)\left\{-\sum_{c}(\operatorname{sign}(c)+s(c))\right\} \otimes \wedge^{d_{s}} \Delta_{s}
$$

The cohomological degree $s(D)=\sum_{c} s(c)$ will be called the height, and the graded degree, equal to the one in the TQFT functor $\mathbf{V}$, up to a shift, will simply be called the degree. The shift from the TQFT degree is prescribed by the integer between braces in such a way that

$$
\mathrm{q}-\operatorname{dim}(G\{i\})=q^{i} \mathrm{q}-\operatorname{dim}(G) .
$$

It is convenient to give a local description of the complex. Here we implicitely extend the definition of $K$ to trivalent diagrams, where only 1-labelled edges are allowed for crossings.

$$
K(\nearrow)=K()()\{-1\} \oplus K(\text { 省 }\{-2\}
$$




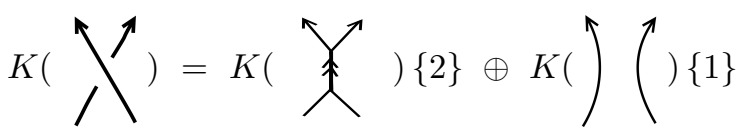

The boundary operator between summands indexed by states $s$ and $s^{\prime}$ is zero unless $s$ and $s^{\prime}$ are different only in one crossing $c$, where $s^{\prime}(c)=s(c)+1$. For a positive crossing (resp. a negative crossing) it is then defined using the TQFT map associated with the cobordism $\Sigma$, (resp. $\Sigma^{\prime}$ ) which are identity outside a neighbourhood of the crossing, and are given by a saddle with 2-labelled membrane, around the crossing $c$ with $s(c)=0, s^{\prime}(c)=1\left(\operatorname{resp} . s(c)=-1, s^{\prime}(c)=0\right)$.

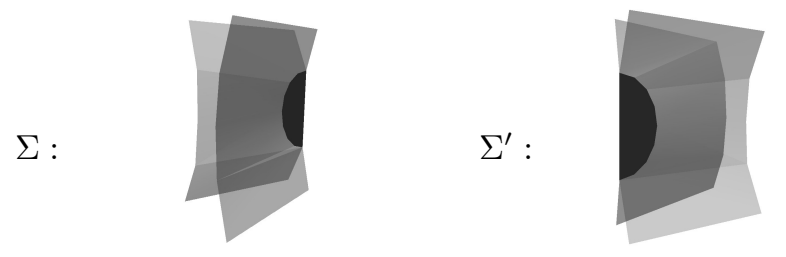

For a positive crossing $c$ :

$$
\delta=\mathbf{V}(\Sigma) \otimes(\bullet \wedge c): \mathbf{V}\left(D_{s}\right) \otimes \wedge^{d_{s}} \Delta_{s} \rightarrow \mathbf{V}\left(D_{s^{\prime}}\right) \otimes \wedge^{d_{s^{\prime}}} \Delta_{s^{\prime}}
$$

For a negative crossing $c$,

$$
\delta=\mathbf{V}\left(\Sigma^{\prime}\right) \otimes\left\langle\bullet, c>: \mathbf{V}\left(D_{s}\right) \otimes \wedge^{d_{s}} \Delta_{s} \rightarrow \mathbf{V}\left(D_{s^{\prime}}\right) \otimes \wedge^{d_{s^{\prime}}} \Delta_{s^{\prime}}\right.
$$

Here $\langle\bullet, c\rangle$ is (the antisymmetrization of) the contraction (using the standard scalar product we understand $c$ as a form).

Theorem 3.1. a) $(K(D), \delta)$ is a graded complex.

b) If the diagrams $D$ and $D^{\prime}$ are related by a Reidemeister move, then there exists an graded homotopy equivalence between the complexes $K(D)$ and $K\left(D^{\prime}\right)$.

c) The graded Euler characteristic is equal to the quantum invariant $\langle D\rangle$, i.e to $q+q^{-1}$ times the Jones polynomial with change of variable $q=-t^{-\frac{1}{2}}$.

We will use the notation $K h(D)$ for the homology of the complex $K$. This theorem says that the isomorphism class of the graded group $K h(D)$ is an invariant of the isotopy class of the corresponding link. We will see later that for a fixed link $L$ (not considered up to isotopy), then $K h(L)$ is well defined.

Proof. We first prove a). The map $\partial$ increases the height by one. The elementary cobordism given by a saddle has Euler caracteristic -1 . The corresponding TQFT map has degree +1 , and the map $\partial$ on the shifted TQFT groups has degree 0 . We want now to compute $\partial \circ \partial$. The possibly non trivial contributions comes from squares corresponding to states $s$ and $s^{\prime \prime}$ identical on all crossings excepted $c_{1}$ and $c_{2}$, where $s^{\prime \prime}(c)=s\left(c_{1}\right)+1$ and $s^{\prime \prime}\left(c_{2}\right)=s\left(c_{2}\right)+1$. We have two intermediate states $s_{1}^{\prime}$ $\left(s_{1}^{\prime}\left(c_{1}\right)=s\left(c_{1}\right)+1\right.$ and $\left.s_{1}^{\prime}\left(c_{2}\right)=s\left(c_{2}\right)\right)$ and $s_{2}^{\prime}\left(s_{2}^{\prime}\left(c_{1}\right)=s\left(c_{1}\right)\right.$ and $\left.s_{2}^{\prime}\left(c_{2}\right)=s\left(c_{2}\right)+1\right)$ given two contributions represented by the same cobordism $\Sigma$ with two saddles (for the TQFT maps, squares commute). Each of them is twisted. We have two check that after twisting the two contributions vanish (squares anticommute) in all cases. If $c_{1}$ and $c_{2}$ are both positive crossings, then we get

$$
\mathbf{V}(\Sigma) \otimes\left(\bullet \wedge c_{1} \wedge c_{2}+\bullet \wedge c_{2} \wedge c_{1}\right)=0
$$




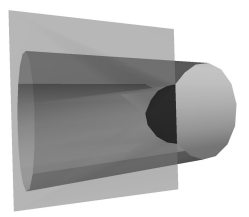

FiguRe 12.

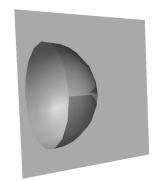

FigURE 13.

If $c_{1}$ and $c_{2}$ are both negative crossings, then we get

$$
\mathbf{V}(\Sigma) \otimes\left(<\bullet, c_{1} \wedge c_{2}>+<\bullet, c_{2} \wedge c_{1}>\right)=0 .
$$

If $c_{1}$ is a positive crossing and $c_{2}$ is a negative crossing, then we get

$$
\mathbf{V}(\Sigma) \otimes\left(<\bullet \wedge c_{1}, c_{2}>+<\bullet \wedge c_{1}>\wedge c_{2}\right)=0 .
$$

The graded Euler characteristic of the complex $K(D)$ satisfies the Jones skein relation (1), and is equal to $q+q^{-1}$ for the trivial diagram. Statement c) follows. In the next subsections we will construct homotopy equivalences for each Reidemeister move, and obtain b).

3.3. Reidemeister move I. We first consider the case of a positive crossing.

$$
K(\uparrow)=[K(\uparrow \bigcirc) \stackrel{\delta}{\longrightarrow} K(\bigcap)]
$$

Recall that the map $\delta$ is equal to $\mathbf{V}\left(\Sigma_{\delta}\right) \otimes(\bullet \wedge c)$ where $\Sigma_{\delta}$ contains a saddle with membrane as depicted in figure 12 . Consider the followings maps.

$$
f: K(\uparrow \bigcirc) \rightarrow K(\uparrow)
$$

is the TQFT map associated with the cobordism in figure 13

$$
g: K(\uparrow) \rightarrow K(\uparrow \bigcirc)
$$

is the sum of the TQFT maps associated with the cobordisms in figure 14.

$$
D: K(\bigcap \rightarrow K(\uparrow \bigcirc)
$$

is equal to $-\mathbf{V}\left(\Sigma_{D}\right) \otimes\langle\bullet, c\rangle$ where $\Sigma_{D}$ is depicted in figure 15

We have that

$$
\delta \circ g=0
$$



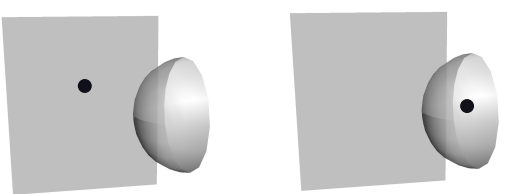

FIGURE 14.

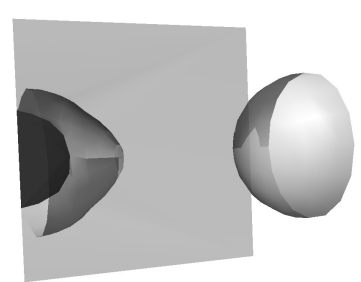

FigURE 15.

Hence $f$ and $g$ define chain maps. We have that

$$
\begin{gathered}
\quad f \circ g=I d \\
I d-g \circ f=D \circ \delta \text { and } \delta \circ D=I d .
\end{gathered}
$$

Hence we have that $D$ is an homotopy between $I d$ and $g \circ f$.

We consider now the case of a negative crossing.

$$
K(\bigcirc)=[K(\supseteqq) \stackrel{\delta}{\longrightarrow} K(\uparrow \bigcirc)]
$$

Here the map $\delta$ is equal to $\mathbf{V}\left(\Sigma_{\delta}\right) \otimes\langle\bullet, c\rangle$ where $\Sigma_{\delta}$ is a saddle. Consider the followings maps.

$$
f: K(\uparrow \bigcirc) \rightarrow K(\uparrow)
$$

is the TQFT map associated with the cobordism in figure 13

$$
g: K(\uparrow) \rightarrow K(\uparrow \bigcirc)
$$

is the sum of the TQFT maps associated the cobordisms in figure 14

$$
D: K(\bigcap \rightarrow K(\uparrow \bigcirc)
$$

is equal to $\mathbf{V}\left(\Sigma_{D}\right) \otimes\langle\bullet, c\rangle$ where $\Sigma_{D}$ is depicted in figure 16

We have that

$$
f \circ \delta=0 \text {. }
$$

Hence $f$ and $g$ define chain maps. We have that

$$
f \circ g=I d
$$




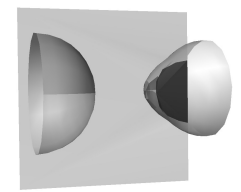

FiguRe 16.

$$
I d-g \circ f=\delta \circ D \text { and } D \circ \delta=I d
$$

Hence we have that $D$ is an homotopy between $I d$ and $g \circ f$.

3.4. Reidemeister $\mathbf{I I}_{+}$. The complexes we want to consider are described in the diagram below.

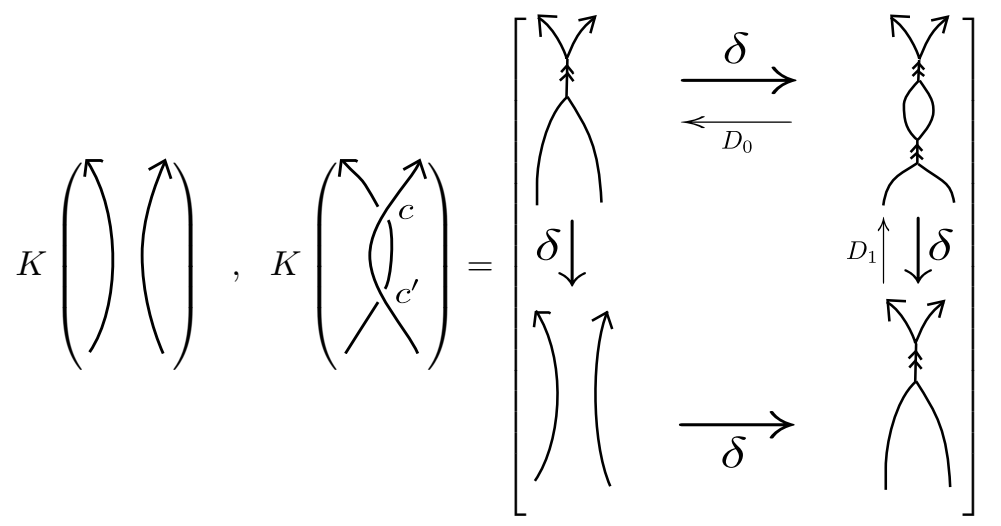

Inverse homotopy equivalences are given by the maps $f$ and $g$ defined below

$$
\begin{gathered}
f=\mathbf{1}_{)} \oplus \mathbf{V}(Z) \otimes\left(\bullet \wedge c \wedge c^{\prime}\right) \\
g=\mathbf{1}_{)} \oplus \mathbf{V}\left(Z^{\prime}\right) \otimes<\bullet, c \wedge c^{\prime}>
\end{gathered}
$$

Here $Z$ and $Z^{\prime}$ are the trivalent surfaces depicted in figure 17 One can check that $\delta \circ f=0$ and $g \circ \delta=0$, so that $f$ and $g$ define chain maps. We have $f \circ g=I d$, moreover there exists $D_{0}, D_{1}$ as depicted in the diagram above such that

$$
\delta \circ D_{1}=I d, D_{0} \circ \delta=I d, g \circ f+D_{1} \circ \delta+\delta \circ D_{0}=I d .
$$

Exercise 3.2. Find $D_{0}, D_{1}$ as expected (hint: use the 4 terms relation in ...).

3.5. Reidemeister II_. Homotopy equivalences for negative Reidemeister move are defined in a similar way. The homotopy equivalence checking rests essentially on the relation in Lemma 2.6a. 

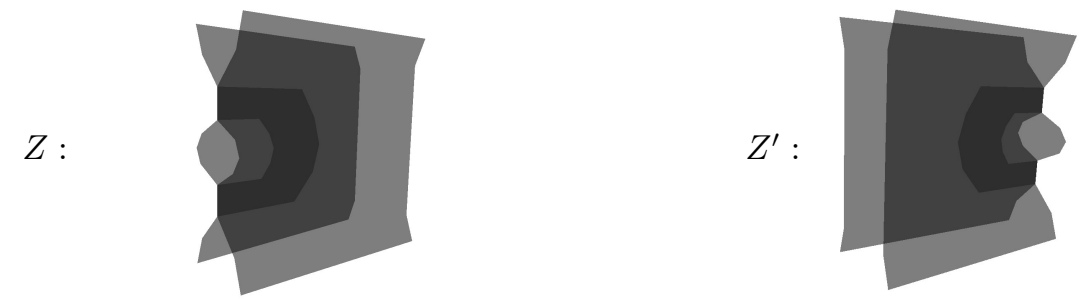

FigURE 17.

3.6. Reidemeister III. We have to consider the complex decomposed as a cube as described below, and the symmetric one.

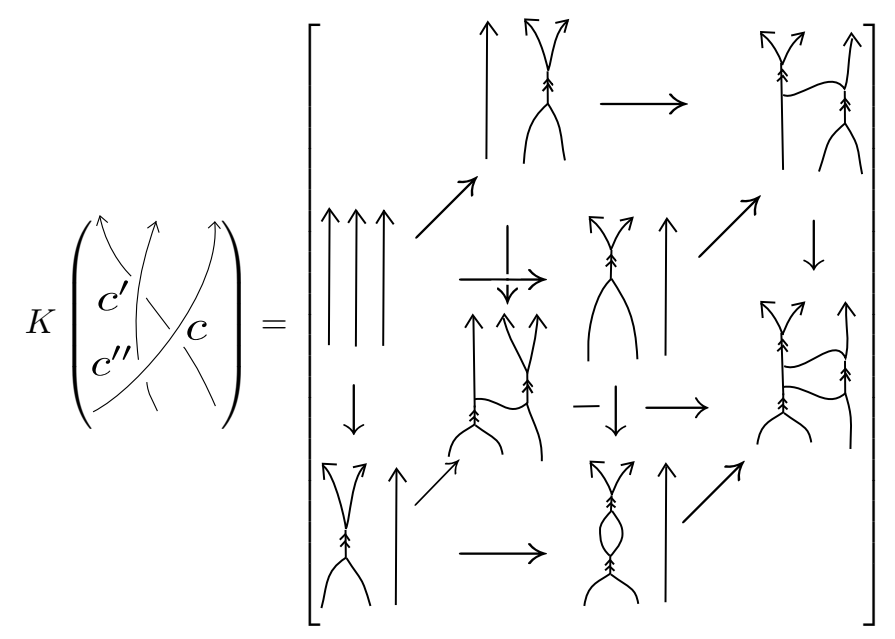

We can find an acyclic subcomplex.

Lemma 3.3. The subcomplex described below is acyclic.

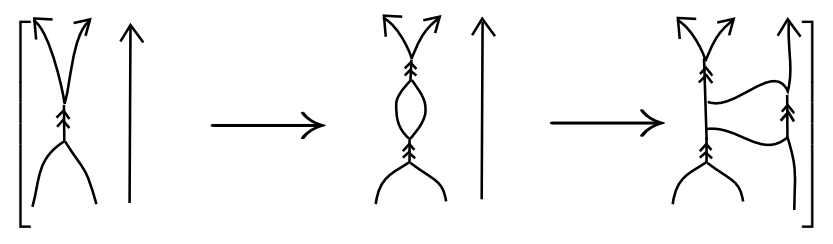

We then apply a Gauss reduction (see e.g. ...) and obtain an homotopy equivalence with a smaller comlex. Note that the above acyclic subcomplex is not a direct summand so that we have to carefully recalculate the boundary map including the action on the twisting determinant. The result is described below Here the boundary maps are given by a saddle with 2 labelled membrane as before twisted 
as indicated near the arrows.

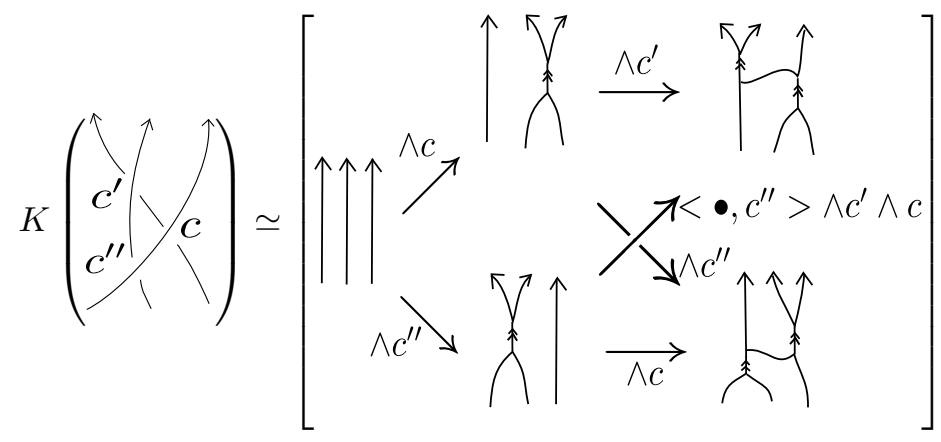

We have

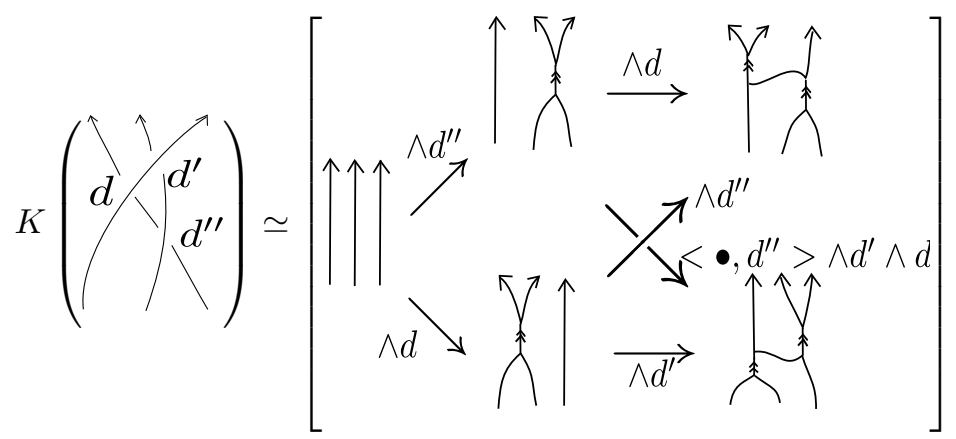

An isomorphism between the two complexes is obtained by using the idendity map on the TQFT modules, and $c \mapsto d^{\prime \prime}, c^{\prime} \mapsto d^{\prime}, c^{\prime \prime} \mapsto d$ on the twisting determinants.

\section{Lee-Rasmussen speCtral Sequence}

Following Lee and Rasmussen, we consider now the Frobenius algebra $\mathbf{A}^{\prime}=\mathbb{Z}[X] / X^{2}-1$, and the associated TQFT functor $\mathbf{V}^{\prime}$. The preceeding construction applies as well, excepted that here the TQFT functor $\mathbf{V}^{\prime}$ is no more graded, but filtered. A generator of a TQFT module $\mathbf{V}^{\prime}(\gamma)$ is given the same degree as before, and $F^{j}\left(\mathbf{V}^{\prime}(\gamma)\right)$ is spanned by generators with degree less or equal to $j$. Observe that a cobordism $\Sigma$ induces a filtered map, with degree equal to $\chi(\Sigma)-2$ (\#points).

We consider the filtered abelian group $K^{\prime}(D)$ defined below, with the same notation as before.

$$
K^{\prime}(D)=\bigoplus_{s} \mathbf{V}^{\prime}\left(D_{s}\right)\left\{-\sum_{c}(\operatorname{sign}(c)+s(c))\right\} \otimes \wedge^{d_{s}} \Delta_{s}
$$

The boundary operator $\partial^{\prime}$ is still defined by twisting the TQFT map associated with a saddle. We denote by $K h^{\prime}(D)$ the homology of this complex.

Theorem 4.1. a) $\left(K^{\prime}(D), \partial^{\prime}\right)$ is a filtered chain complex.

b) If the diagrams $D$ and $D^{\prime}$ are related by a Reidemeister move, then there exists a filtered homotopy equivalence between the complexes $K^{\prime}(D)$ and $K^{\prime}\left(D^{\prime}\right)$.

c) There exists a spectral sequence whose second page is the homology of our complex $K$

$$
E_{2}^{i, j}(D)=K h^{i-j, j}(D)
$$

which converges to $K h^{\prime *}(D)$. 
Proof. a) and b) are proved as before. Statement c) follows from standard facts with filtered chain complexes.

The theorem shows that all the pages with index greater or equal to 2 are invariants of the link. For Khovanov original homology, it was proved by Lee [Lee that the limit depends only on the number of components. Rasmussen Ras was able to extract a lower bound for the slice genus and to use it to give a combinatorial proof of Milnor conjecture on the slice genus of torus knots.

We will compute our oriented version of Lee-Rasmussen homology over $\Lambda=\mathbb{Z}\left[\frac{1}{2}\right]$ using the Karoubi completion method of Bar-Natan and Morrisson [BM]. We denote by $K h^{\prime}(D, \Lambda)$ the homology of $K^{\prime}(D) \otimes \Lambda$.

Theorem 4.2. For a link diagram $D$ with $m$ components, $K h^{\prime}(D, \Lambda)$ is a free $\Lambda$-module of rank $2^{m}$, with a canonical basis indexed by maps $\epsilon: \pi_{0}(D) \rightarrow\{ \pm 1\}$.

Proof. The algebra $\mathbf{A}^{\prime}$ contains the minimal idempotents

$$
\pi_{ \pm}=\frac{1 \pm X}{2} .
$$

Using these idempotents we extend the TQFT functor $\mathbf{V}^{\prime}$ to an extended trivalent category where 1-labelled edges or faces may be colored with $\pi_{ \pm}$. If 1-labelled edges in a trivalent graph $\gamma$ are colored with a sequence of signs denoted by $\epsilon$, then we obtain an object $\gamma(\epsilon)$ whose associated module is the image of the obvious projector $\pi_{\epsilon} \in \mathbf{V}^{\prime}(\gamma)$, associated with $\epsilon$. The relation in Lemma 1.3 a) still hold for the functor $\mathbf{V}^{\prime}$. We deduce that the module $\mathbf{V}^{\prime}(\gamma)$ is zero if signs agree on the two 1-labelled edges adjacent to a vertex. For an alternating sign assignement $\epsilon$, the module $\mathbf{V}^{\prime}(\gamma(\epsilon))$ has rank one with a basis represented by a trivalent surface whith only discs (without points) as 2-cells. The complex $K^{\prime}(D)$ which was a sum indexed by states, is now decomposed into a sum indexed by colored states (states with coloring of all arcs). The boundary map can be computed locally. The TQFT map associated to a saddle is zero unless all colors coincide on the arcs belonging to the same 1-labelled component. In the remaining case, this TQFT map is an isomorphism. We obtain a deformation retract on a subcomplex $K^{\prime \prime}(D)$ where the boudary map is zero. Locally, i.e. for a crossing, the subcomplex $K^{\prime \prime}(D)$ is described below.

\section{DESSIN}

For a generator, the signs associated with arcs belonging to the same component of the represented link are the same. Moreover for an assignment of signs on the components, the state of each crossing is determined so there is a unique corresponding generator.

\section{Functoriality}

Extension of Khovanov homology to link cobordisms and functoriality up to sign was conjectured by Khovanov and established by Jacobsson Jac and Khovanov [Kh3, and Bar-Natan [BN2]. The sign ambiguity was carried over by ClarkMorrison-Walker [CMW]. In this section we show that our construction has a strictly functorial extension.

A movie description of a cobordism is a generic projection in $[0,1] \times \mathbb{R}^{2}$ of a smooth surface in $[0,1] \times \mathbb{R}^{3}$. Generically a movie decomposes into elementary ones which either describe a Reidemeister move or glue an handle to the surface. To 
each Reidemeister type movie we associate the corresponding homotopy equivalence, and to each handle addition we associate the chain map associated with the corresponding TQFT map. We then associate to a movie the composition of these elementary chain maps.

Theorem 5.1. a) Let $L \subset S^{3}$ be a link represented by diagrams $D$ and $D^{\prime}$, then the homology isomorphism associated to a sequence of Reidemeister moves is canonical, i. e. $K h(L)$ is well defined.

b) Let $C \subset[0,1] \times \S^{3}$ be a smooth cobordism between the links $L$ and $L^{\prime}$. The homology map $K h(L) \rightarrow K h\left(L^{\prime}\right)$ induced by a movie description of $C$ only depends of the isotopy class of $C$ rel. $L \times\{0\} \cup L^{\prime} \times\{1\}$, and $K h$ extends to a functor on the embedded cobordism category.

Remark 5.2. Note that here we consider fixed links, and not links up to isotopy. It was observed by Jacobson that Khovanov homology do have monodromy.

Proof. In a) we may consider the link in $\mathbb{R}^{3}$ as well. Then a diagram is associated with a generic projection on an oriented plane, and is parametrized by a point in $S^{2}$. A generic loop in $S^{2}$ result in a finite sequence of Reidemeister moves, and induces an homology isomorphism. An homotopy to the trivial loop can be obtained via elementary homotopies corresponding to Reidemeister type movie moves. So statement a) results from Lemma 5.3 . More generally movie moves generate isotopies of embedded surfaces whence staement b).

Lemma 5.3. For each movie move the two induced homology maps coincide.

Proof. We first show projective functoriality, using a simplicity argument of BarNatan. Then we consider the degenerate Lee theory. For a given movie move, the sign is the same for the degenerate theory. We have seen that $K h^{\prime}(L)$ has a distinguished element associated with the assignement of a positive sign on all components. We can see that the map associated with an elementary movie respects this canonical element.

\section{REFERENCES}

[BN] Dror Bar Natan, On Khovanov's categorification of the Jones polynomial, Algebraic and Geometric Topology 2 (2002) 337-370.

[BN2] Dror Bar Natan, Khovanov's homology for tangles and cobordisms, Geometry and Topology 9 (2005) 1443-1499.

[BM] Dror Bar Natan and Scott Morrison, The Karoubi Envelope and Lee's Degeneration of Khovanov Homology, math.GT/0606542

[BHMV] C. Blanchet, N. Habegger, G. Masbaum, P. Vogel, Topological Quantum Field Theories derived from the Kauffman bracket, Topology 34 (1995), 883-927.

[CC] Carmen Livia Caprau, sl(2) tangle homology with a parameter and singular cobordisms, Algebraic \& Geometric Topology 8, 729-756 (2008).

[CMW] Davis Clark, Scott Morrison and Kevin Walker, Fixing the functoriality of Khovanov homology, math.GT/0701339

[Jac] Magnus Jacobson, An invariant of link cobordisms from Khovanov homology, Algebr. Geom. Topol. 4 (2004) 1211-1251.

[Ko] Kock J, (2003) Frobenius Algebras and 2D Topological Quantum Field Theories. LMS Student Texts 59, Cambridge University Press.

[Kh] Mikhail Khovanov, A categorification of the Jones polynomial, Duke Math. J. 101, No.3, 359-426 (2000).

[Kh2] Mikhail Khovanov, A functor-valued invariant of tangles, Algebr. Geom. Topol. 2 (2002) $665-741$. 
[Kh3] Mikhail Khovanov, An invariant of tangle cobordisms , Trans. Amer. Math. Soc. 358 (2006), 315-327.

[Lee] Eun Soo Lee, An endomorphism of the Khovanov invariant, Adv. Math. 197, No.2, 554-586 (2005).

[Ras] Jacob Rasmussen, Khovanov homology and the slice genus, math.GT/0402131 